\title{
Analytical ETL/EML Layer Investigation of Blue OLEDs
}

\author{
Andreas Graff ${ }^{1}$, Frank Altmann ${ }^{1}$, Andrzej Dzwilewski ${ }^{2}$ and Bert Freitag ${ }^{3}$. \\ 1. Fraunhofer Institute for Mechanics of Materials, Halle, Germany. \\ 2. Novaled GmbH, Dresden, Germany. \\ ${ }^{3 .}$ FEI Company, Eindhoven, The Netherlands.
}

Organic light emitting diodes (OLEDs) based display technologies are getting more and more important for smartphones, laptops and televisions. OLED devices consist of thin organic layer stacks between two electrodes. Located around an emission layer, where the radiative recombination of electrons and holes takes place, are layers for the transport and blocking of charge carriers. Thickness, composition and structure of the layers influence color, efficiency and lifetime of OLED devices. Characterization for process qualification and failure analysis of the OLED stacks is pivotal to improve these properties. The challenge in this material is to prepare the relevant area, obtain contrast to resolve the layers and not damage the layers even under high beam current analytical experimental conditions. In this paper the complete workflow from preparation of a sample from a working mobile phone to examination of critical organic LED layers is presented.

We used a focused ion beam (FIB) for the preparation of a side specific electron transparent samples (100nm) of a blue OLED of a mobile phone display using a FEI Versa 3D. TEM investigations were performed in an image corrected microscope (Titan G2 60-300, FEI) equipped with an image filter, a high brightness field emission gun (X-FEG) and a dedicated EDS detector (SuperX) [1] for high sensitivity to prevent damage of such delicate material. TEM investigations were done at $80 \mathrm{kV}$ and $300 \mathrm{kV}$ in TEM and STEM with variations of the beam intensity in order to optimize the bright field contrast of the different OLED layers and to minimize electron beam damage during XEDS mapping.

OLED Reference samples (to verify the process on a known layer stack) and display pixels were chosen to prepare TEM lamellas by FIB using the clip holder lift out technique [2]. The lamellas, fixed in a special TEM grid, are thinned from both sides finishing with low voltage FIB polish to reduce sidewall artefacts thus reaching a thickness of $100 \mathrm{~nm}$ without much bending of the TEM cross section (Fig.1 right). TEM and STEM images at 80 $\mathrm{kV}$ show that the uppermost organic layers the electron transport layer (ETL), the emission layer (EML) and the electron blocking layer (EBL) could be distinguished due to their different density (Fig.2). Even more information can be gained by the use of XEDS mapping. The highly sensitive EDS detector allows the collection of a reasonable amount of element specific X-rays in a short period of time and more important less beam current can be used. Element distributions can be acquired in minutes before the organic sample changes its morphology. Figure 3 shows the element maps of nitrogen, oxygen, aluminum and indium which were taken within only $14 \mathrm{~ms}$ per pixel. The organic layers differ in carbon, nitrogen and oxygen content and can be distinguished in the XEDS maps and intensity profiles. Impurities like fluorine can be found near the In-oxide anode (hole transport layer, HTL, which is usually doped with strong p-dopants frequently containing Fluor groups) but also in the lower part of the emission layer, which can influence the performance of these layers. Oxidation of the aluminum interface to the organic layers is also visible.

This study proves that FIB preparation in combination with low voltage TEM imaging and XEDS mapping provides critical structural and chemical information which makes it a valuable tool for the investigation of functional organic layers in electronic devices.

References:

[1] L.J. Allen et al, MRS Bulletin 37 (2012) 47-52

[2] F. Altmann et al, Microsc. Microanal. 17 (2011) p. 627. 

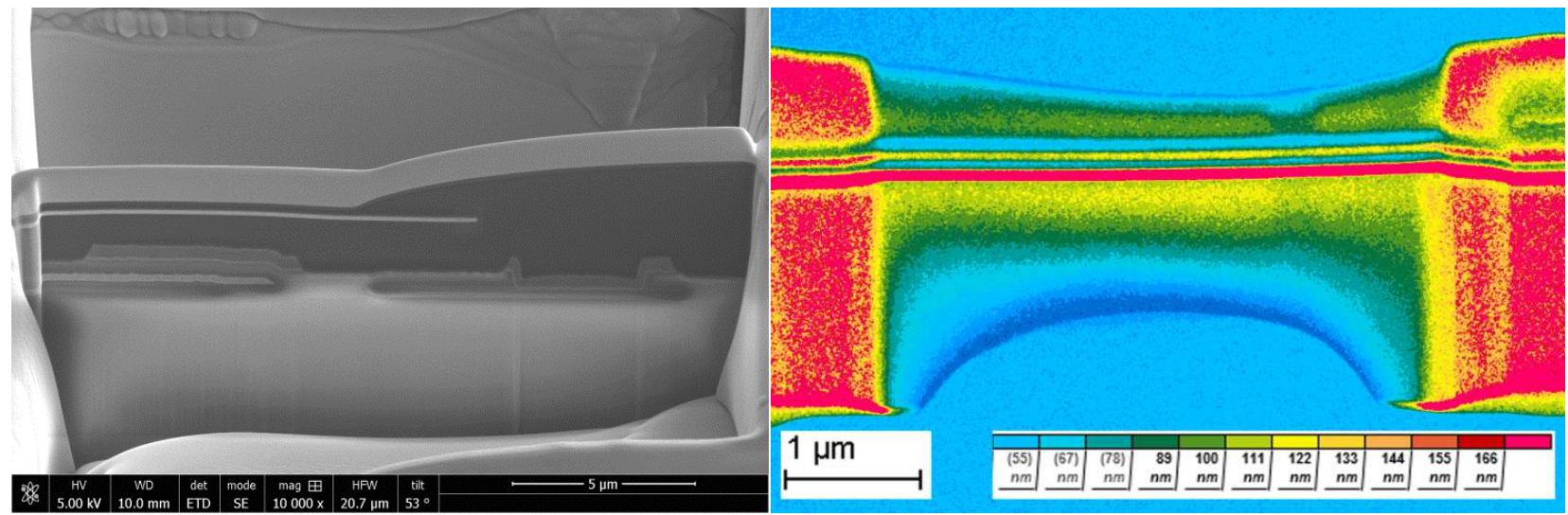

Figure 1. Left: FIB cross section of an OLED device. Right: Thickness map out of an SEM image of the final FIB prepared TEM cross section in a magazine holder.
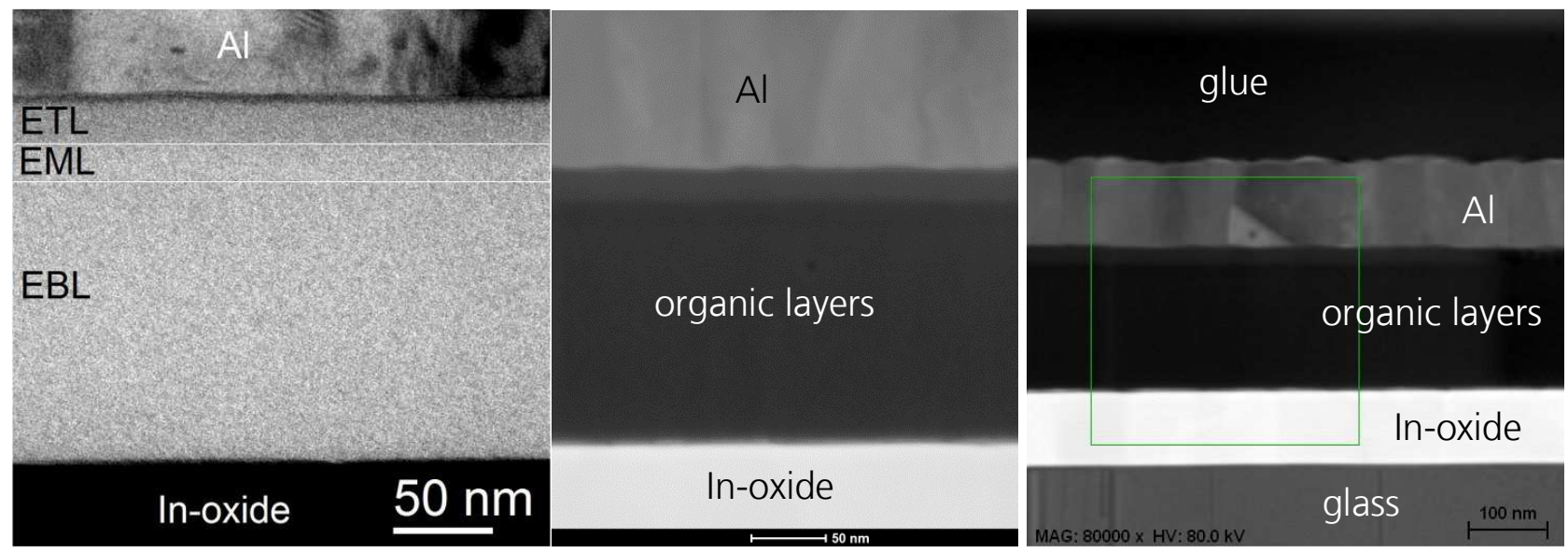

Figure 2. Left: TEM bright field image of the OLED layer stack. Middle: STEM HAADF image of the organic layers. Right: STEM HAADF image of the layer stack with region of XEDS mapping marked.
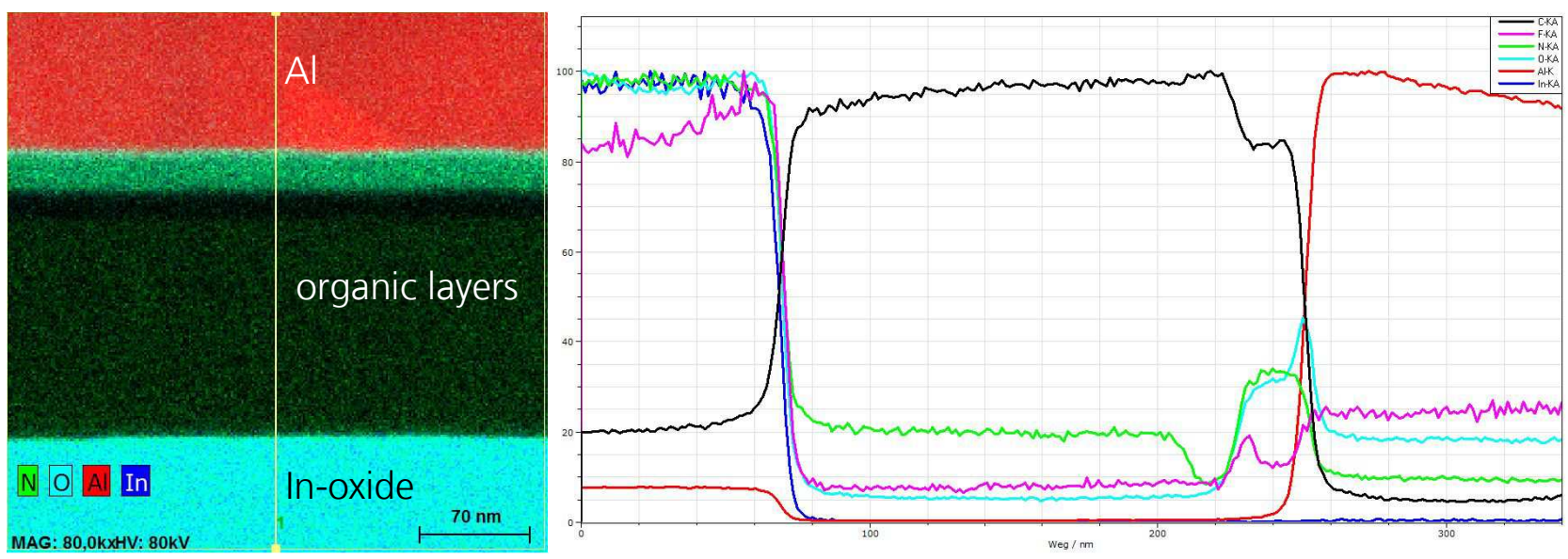

Figure 3. Left: Color coded results of the XEDS mapping of the OLED layer stack. Right: Line profile out of the EDXS mapping across the layer stack. 